\title{
A Numerical Study of Air Conditioning Cycle Using Alternative Refrigerants
}

\author{
ByeongSu Kim¹, Yongchan Kim ${ }^{2}$ \\ ${ }^{1}$ Graduate School of Mechanical Engineering, Korea University \\ Anam-ro 145, Seongbuk-gu, Seoul, Republic of Korea \\ pnu-mecha99@korea.ac.kr \\ ${ }^{2}$ Department of Mechanical Engineering, Korea University \\ Anam-ro 145, Seongbuk-gu, Seoul, Republic of Korea \\ yongckim@korea.ac.kr
}

\section{Extended Abstract}

With strong F-Gas regulation due to global warming, there is a growing demand for new alternative refrigerants. Thus, a large number of alternative refrigerants are currently being developed. According to the United Nations Environment Program's (UNEP) Technology and Economic Assessment Panel (TEAP) report [1], in 2016, more than 80 alternative refrigerants have been evaluated and developed around the world. However, it is difficult to judge which of the many alternative refrigerants can be applied to the product, or which of them shows most optimal performance, because of the lack of performance comparison or simulation results among the refrigerants.

In this study, the most optimal alternative refrigerants at various operating conditions are suggested, based on the numerical study on the performances of refrigeration cycles that use the alternative refrigerants as the working fluids. Numerical studies were performed on approximately 80 refrigerants, which include the existing refrigerants and the alternative refrigerants that have known compositions according to the TEAP report. The refrigerant properties were applied using the REFPROP program of the National Institute of Standards and Technology (NIST).

The state of the refrigerant discharged from the compressor was calculated based on the specifications provided by the manufacturer, such as the refrigerant flow rate, power consumption, isentropic efficiency. They were measured according to the evaporating/condensation temperatures and the compressor operating frequency. The operating frequency of the compressor was adjusted so that the calculated capacity was within $\pm 1 \%$ of the target capacity. Condenser and evaporator performances were calculated by the segment by segment method, which calculates the amount of heat on the refrigerant side and the air side by dividing the whole heat exchanger into several segments and using each segment as the control volume. In this case, the outlet condition of the previous segment is substituted as the inlet condition of the next segment, and single phase or two phase heat transfer and pressure drop correlations are applied according to phase of the segment inlet refrigerant. In the air side, the well-known heat transfer and pressure drop correlations are applied according to the types of heat transfer fins (Plate, Wavy, Slit and Louver). The condenser performance and outlet conditions are calculated using the refrigerant flow rate calculated by the compressor, and the iterative calculation is performed while varying the condensation temperature until the target subcooling and the calculated subcooling match. Then, the evaporator performance and the outlet condition are calculated through the evaporator calculation, and the iterative calculation is performed while changing the evaporation temperature until the target superheat and the calculated superheat match. Finally, when the calculation is completed, the target subcooling is changed and the iterative calculation is performed again to find the best operation condition that yields the maximum efficiency.

As a result, we were able to find a number of possible alternative refrigerants that could replace refrigerants such as the R410A currently in use through simulation.

\section{References}

[1] B. Maranion, et al, (2015), Decision XXVI/9 Update Task Force Report Additional Information on Alternatives to Ozone-Depleting Substances. [Online]. Available: http://conf.montreal-protocol.org/meeting/mop/mop27/presession/Background\%20Documents\%20are\%20available\%20in\%20English\%20only/TEAP_Task-ForceXXVI-9_Update-Report_September-2015.pdf 